\title{
Double-blind Clinical Evaluation of UCB 1549 in Treatment of Parkinson's Disease
}

\author{
R. R. STRANG,* M.D.
}

Brit. med. F., 1966, 2, 1112-1113

The occurrence of intolerable side-effects at a clinically effective dosage level, a loss of therapeutic efficacy with the passage of time, and failure to respond to specific drugs are some of the more cogent reasons for the development of new and effective anti-Parkinson medicaments.

The need is particularly urgent, since it has become clear that pallido-thalamic surgery is largely selective in its effects, and has not, or only slightly, reduced drug-dependence for many patients. Less than $5 \%$ of those seen post-operatively had discontinued drug therapy, and in almost all of these it was for only a relatively short time. A not inconsiderable number require a more intensive postoperative medical regimen, including periods of regular and graduated physical therapy. A marked reduction in postoperative drug therapy probably contributes to, and may, in some cases at least, be largely responsible for, the frequently observed exacerbation or precipitation of symptoms on the contralateral side after surgery.

In addition, many patients are too old or are otherwise unsuitable for surgical treatment. Approximately one-third of those under the age of 67 referred for operative evaluation are rejected as a result of one or more of the general clinical, neurological, radiological, electroencephalographic, or more specialized examinations. In addition, recurrences, the incidence of which increases with the passage of time, necessitate concentrated medical treatment at a preoperative or higher level of intensity.

Any drug which can maintain a significant degree of clinical improvement in at least $30 \%$ of patients for a minimum period of three to four months is a worth-while addition to the overall therapeutic armamentarium of Parkinsonism.

\section{Pharmacology}

UCB 1549 (2-(2-dimethylethoxy) ethanol-1-phenyl-cyclopentyl-carboxylate) has been shown to possess a strong central and a relatively weak peripheral anticholinergic action in animals. ${ }^{1}$ It is actively spasmolytic, though significantly less so than atropine. The drug has virtually no antihistaminic properties.

It was well tolerated by rats in oral doses of $165 \mathrm{mg} . / \mathrm{kg}$., and the L.D. ${ }_{50}$ in these animals averaged $42 \mathrm{mg} . / \mathrm{kg}$. intravenously and $1.27 \mathrm{~g} . / \mathrm{kg}$. orally. The central nystagmus produced by the electrical stimulation of the diencephalon of rabbits was depressed by raising the threshold of excitability.

The drug, which is available in scored 50-mg. tablets, has the following structural formula:<smiles>CN(C)CCOCCOCC(=O)C1CCCC1</smiles>

\section{Clinical Material and Method}

The age-and-sex distribution of the 100 consecutively examined unselected patients with non-chemical Parkinsonism who participated in the trial was as follows:

$\begin{array}{ccccc}\text { Age (years) } & & \text { Males } & & \text { Females } \\ 28-49 & \ldots & 15 & \ldots & 10 \\ 50-64 & \ldots & 23 & \ldots & 16 \\ 65-77 & \ldots & 22 & \ldots & 14\end{array}$

Twenty-four patients were classified as post-encephalitic, 23 as arteriosclerotic, and 53 as cases of "idiopathic" Parkinsonism. Alternate patients were given envelopes containing the same dosage instructions, and either UCB 1549 or identically prepared placebo tablets. The distribution sequence varied with each group of 10 consecutive patients examined.

At the end of the two-month double-blind trial patients receiving placebo tablets were given UCB 1549 with the same dosage instructions, and all patients were finally examined at the end of a five-month observation period of drug therapy, or earlier if the treatment was discontinued. The key to the double-blind trial was held by an independent observer. Close contact with the patients was kept throughout the trial, and advice was often sought regarding the dosage increases, the occurrence of side-effects, and the clinical effects.

The initial dosage recommended was half a tablet $(25 \mathrm{mg}$.) daily for three days, followed by half-tablet increases every second day until a satisfactory clinical response was obtained, or until the daily total reached six tablets. If this dosage failed to produce a significant clinical effect the drug was discontinued after 10 days. According to individual preference, UCB 1549 was taken three or four times a day with meals or a light snack. If troublesome and persistent side-effects occurred the dose was reduced to a non-toxic level for 7 to 10 days, when careful, wellspaced increases were again tried if further clinical improvement was thought possible.

Thirty-four of the patients receiving UCB 1549 and 32 of those given a placebo were taking one or more specific antiParkinson drugs when first seen, and the dosages of these were continued unchanged throughout the trial.

All examinations were carried out by me, and the symptoms and signs were evaluated clinically, both objectively and subjectively, and the results graded as a percentage, in units of 10 , from $0 \%$ (normal) to $100 \%$ (maximum severity). For the purpose of clinical assessment, an improvement of less than $10 \%$ was not regarded as "significant." This figure is arbitrarily chosen because of the natural variability of the illness itself, and because of the impossibility of accurately grading such slight changes. The latter difficulty may be partially overcome by rejecting equivocal differences, basing the results on the average change recorded in a number of joints for both tremor and rigidity, and by the continuous examination of large numbers of patients with Parkinson's disease. To increase the degree of accuracy and consistency commonly used timed performance tests were carried out as a measure of the patient's "functional capacity," the results affording a reasonably accurate and practical control of the clinical findings. A dynamometer was used to record the strength of the hand grips.

Laboratory examinations (routine blood, urine, and liver studies) were carried out on 10 patients.

\section{Clinical Results}

At the end of two months, when the double-blind trial was concluded, 26 of the 50 patients taking UCB 1549 had main-

\footnotetext{
* Present address : 67 Nicholson Street, Chatswood, Sydney, New South Wales, Australia.

1 The pharmacological data were kindly supplied by U.C.B.-Pharmaceutical Division, Brussels, Belgium, manufacturers of the drug.
} 
tained a significant degree (at least $10 \%$ ) of clinical improvement. Of the patients receiving the placebo 30 were not significantly changed, and 20 , including 15 who were not being treated when first seen, had clinically regressed. Of the 24 patients unimproved by UCB 1549 six discontinued the drug because of side-effects, five became worse, and 13 showed no significant response.

At the end of five months 44 of the 100 patients had maintained a significant degree of improvement of the functional capacity, as measured by the timed performance tests. The results of treatment and the grade of improvement at this time are summarized in the Table. In no instance did the recorded improvement of a symptom or sign exceed $50 \%$ when the final evaluation was made, and an improvement of more than $30 \%$ occurred almost exclusively in patients with mild to moderate degrees of incapacity.

Results of Treating 100 Cases of Parkinson's Disease with UCB 1549

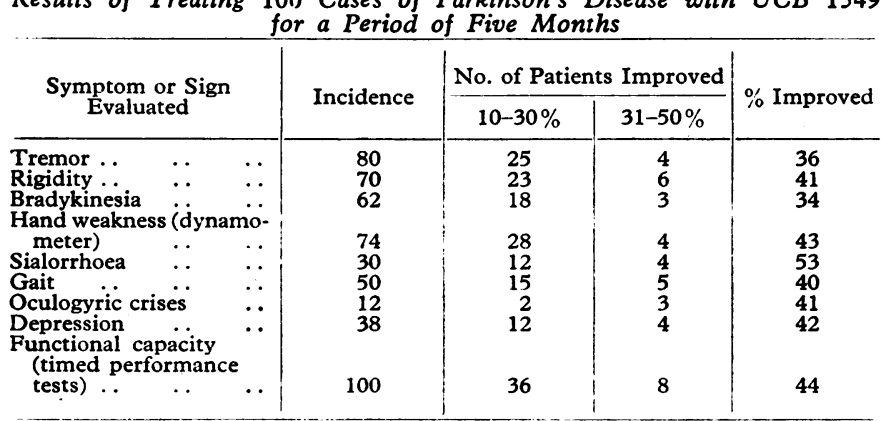

Sialorrhoea, sweating attacks, and oculogyric crises responded satisfactorily to the drug, and in three cases with oculogyric crises the control afforded was particularly good. The improvement of depression was thought to be secondary in all cases, and a significant primary psychostimulating effect was not observed. On the contrary, five of the patients unimproved by the drug became mildly to moderately depressed, although readily conceding that clinical regression had not occurred.

The idiopathic group of patients responded to UCB 1549 most satisfactorily and the arteriosclerotic group least satisfactorily. The latter group had the lowest degree of drug tolerance and the post-encephalitic group the greatest.

Side-effects troublesome enough to require withdrawal of the drug occurred in 16 of the 100 patients. The most commonly observed side-effects were dizziness, blurred vision, palpitations, dryness of the oropharyngeal mucosa, constipation, apathy, and tiredness. Less common were headache, angor animi, akathisia, confusion in two cases, inability to urinate in two, and an erythematous skin rash in one case.

At the end of five months 58 patients were still taking the drug ; the average daily dose was five tablets in patients up to the age of 60 , and 3.5 tablets in those aged 61 to 77 .

A pilot study of 15 hospital inpatients revealed that the clinical effect of a single $50-\mathrm{mg}$. dose had a latency of onset ranging from 15 to 60 minutes, with an average of 30 minutes, and a duration of action ranging from half to three and threequarter hours. Additional improvement rarely followed doses above six tablets a day, and such levels were poorly tolerated by at least $30 \%$ of the patients.

Of the 44 patients responding satisfactorily to UCB 1549 at the end of five months $27(41 \%$ of 66$)$ were taking one or more anti-Parkinson drugs and $17(50 \%$ of 34$)$ were untreated when first seen.

No significant alteration occurred in any of the control laboratory examinations.

\section{Discussion}

The clinical improvement produced in 26 out of 50 patients at the end of two months and in 44 out of 100 patients at the end of five months indicates that UCB 1549 is a worth-while addition to the drug armamentarium of Parkinsonism.

Side-effects are slightly more frequent from UCB 1549 than from other commonly used anticholinergic medications, with the exception of Artane (benzhexol hydrochloride). Advice to the patients was often necessary in the early stages of treatment, and it soon became apparent that in a number of instances better results and greater tolerance could be obtained by third or fourth daily half-tablet increases. In a few elderly patients who had the poorest drug tolerance one-quarter tablet increments were tried. During the trial, and since its conclusion, seven patients who discontinued UCB 1549 because of poor tolerance or inadequate effect have been more carefully restarted on the drug, with a satisfactory clinical response. The present procedure is to recommend, initially, third or fourth daily halftablet increases for patients over the age of 60 , and half-tablet second daily increases for those aged 60 or under.

The best results were obtained in the group having idiopathic Parkinsonism. These patients were the youngest in the trial and had the shortest histories and the least severe forms of the disease.

UCB 1549 combined quite well with other anti-Parkinson drugs, and the best results were obtained with Disipal (orphenadrine hydrochloride), Tremonil (methixene hydrochloride), and Phenoxene (chlorphenoxamine hydrochloride). Of the 66 patients on combined therapy 10 stated that UCB 1549 was the best medication they had received, and five responded well, although they had previously been very difficult to treat.

When tremor was very troublesome the most satisfactory control was afforded by taking the drug three times a day with meals, whereas an additional dose was often necessary to produce the best results when rigidity and/or bradykinesia were the main complaints.

\section{Summary}

UCB 1549 is an effective anti-Parkinson drug, having maintained in the present trial a significant degree of clinical improvement in 44 out of 100 patients observed for a period of five months.

Side-effects are occasionally troublesome, and, particularly early in treatment, small well-spaced dosage increases may be necessary to produce tolerance and to achieve an optimal clinical effect, especially in the older age groups.

UCB 1549 is useful in all forms of the disease, and combines well with other specific anti-Parkinson drugs.

It is most effective against rigidity, and least so against bradykinesia. The improvement regarding tremor occupies an intermediate position, and quantitatively is quite satisfactory. 